\title{
Aproximación al problema contemporáneo de la Energía y el Desarrollo Sostenible con Técnicas de Gamificación
}

\section{Sergio Gallardo ${ }^{a}$, José Felipe Villanueva ${ }^{b}$, María Lorduyc, Belén Juste ${ }^{d}$}

${ }^{a}$ Dpto. de Ingeniería Química y Nuclear, Universitat Politècnica de València, Spain, email: sergalbe@iqn.upv.es; 'b Dpto. de Ingeniería Química y Nuclear, Universitat Politècnica de València, Spain, email:jovillo0@upvnet.upv.es; 'Instituto Universitario de Seguridad Industrial, Radiofísica y Mediambiental (ISIRYM), Universitat Politècnica de València, Spain, email: maloral@upv.es; ${ }^{\mathrm{d}}$ Dpto. de Ingeniería Química y Nuclear, Universitat Politècnica de València, Spain, email: bejusvi@iqn.upv.es

\begin{abstract}
In recent years, the need to explore new teaching strategies has been proven, which encourages a more active role on the part of the students and which favors the motivation to learn. In this framework, it is worth highlighting methodologies such as gamification. In the techniques of gamification, game mechanics are applied in areas that are normally not playful, enhancing the involvement of students through rewards.

This methodology is being applied in the subject "Energy and Sustainable Development" of the Master's Degree in Industrial Engineering (MUII) of the Technical School of Industrial Engineers (ETSII) of the Universitat Politècnica de València (UPV), mainly aimed at small groups of exchange students.

In this subject, with an eminently practical approach, several gamified activities are proposed for learning of contents related to renewable energies: It is about carrying out the energy planning of an imaginary country that includes a series of economic and environmental restrictions. Depending on the results and their argumentation, "stars" are awarded, which allows students to acquire learning stimulated by reasoning and the added motivation of the game.
\end{abstract}

Keywords: Gamification, Specific competencies, Transversal competencies, Master Engineering.

\section{Resumen}

En los últimos años se ha comprobado la necesidad de explorar nuevas estrategias de enseñanza, que potencien un rol más activo por parte de los alumnos y que favorezcan la motivación por aprender. En este marco, cabe destacar metodologías como la gamificación. En las técnicas de ludificación o gamificación se aplican mecánicas de juego en ámbitos que normalmente no son lúdicos, potenciándose la involucración del alumnado mediante recompensas.

Esta metodología se está aplicando en la asignatura "Energy and Sustainable Development” del Máster Universitario de Ingeniería Industrial (MUII) de la Escuela Técnica Superior de Ingenieros Industriales (ETSII) de la Universitat 
Politècnica de València (UPV), fundamentalmente dirigida a grupos reducidos de alumnos de intercambio.

En esta asignatura, de enfoque eminentemente práctico, se plantean diversas actividades gamificadas para el aprendizaje de contenidos relacionados con las energías renovables: se trata de realizar la planificación energética de un país imaginario al que se le incluye una serie de restricciones económicas y medioambientales. En función de los resultados y su argumentación se otorgan "estrellas", lo que permite alumno adquirir un aprendizaje estimulado por el razonamiento y la motivación añadida del juego.

Palabras clave: Gamificación, Competencias específicas, Competencias transversales, Máster en Ingeniería.

\section{Introducción}

El Espacio Europeo de Educación Superior (EEES) ha propiciado la aparición y desarrollo de nuevas metodologías de trabajo dentro y fuera del aula para potenciar un rol más activo por parte de los alumnos. Este nuevo enfoque obliga a abandonar la tradicional zona de confort tanto de los profesores como de los alumnos. En el caso de los profesores se debe producir un cambio de mentalidad para renunciar al esquema actual de transmisión de conocimientos y asimilar nuevas metodologías de trabajo con el fin de proporcionar herramientas a los alumnos para que puedan adquirir conocimientos de manera más autónoma. La motivación por aprender es esencial (Romero y Pérez Ferra, 2009), ya que es una de las variables que más incidencia tiene en el rendimiento del alumnado. Los actuales resultados en los indicadores de muchas titulaciones evidencian la necesidad de explorar nuevas estrategias de motivación por parte del profesorado. Este contexto exige modificar la forma en la que se presenta la información al alumnado y, por lo tanto, implica un cambio en la relación entre los profesores y alumnos. En este marco, metodologías como la clase inversa (o flipped classroom) y la gamificación están comenzando a hacerse un hueco en la docencia universitaria.

En las técnicas de ludificación o gamificación se aplican mecánicas de juego en ámbitos que normalmente no son lúdicos (Deterding et al., 2011, Llagostera, 2012, Werbach, 2014 y Attali y Arieli-Attali, 2015). Muchos aspectos del juego y los propios juegos son muy propicios para el aprendizaje (Bruder, 2015). La gamificación en la educación pretende potenciar la acción, promover el aprendizaje y resolver problemas (Kapp, 2012). Sin embargo, hasta la fecha, las experiencias y resultados publicados sobre los beneficios e inconvenientes de utilizar esta técnica son estudios puntuales realizados en un único curso académico y con equipos de alumnos en muchas ocasiones también reducidos (Urquidi y Calabor, 2014, Ibañez, 2015, Castilla et al., 2013 y Monguillot et al., 2015).

Mediante las actividades basadas en juegos se pueden potenciar nuevos hábitos de trabajo más motivadores, potenciando la concentración y el esfuerzo (Deterding et al., 2011). ¿Cómo transformar una actividad formativa convencional en una actividad gamificada? A priori, se puede caer en el error en transformar la actividad en un juego, trivializándolo y obviando el fin último de aprendizaje. Resulta indispensable ser conscientes de lo que implica la gamificación,

(c) ) EY-NC-ND 2018, Universitat Politècnica de València

Congreso IN-RED (2018) 
cuáles son sus ventajas e inconvenientes, de qué herramientas dispone el docente para llevar a cabo este tipo de actividades y cómo debe presentar la actividad al alumnado.

En principio, cualquier actividad puede ser gamificada si se cumplen las siguientes premisas: la actividad puede aprenderse, las acciones de los usuarios se pueden medir y, existe una realimentación al mismo (Gónzalez y Mora, 2015). Las motivaciones que se pueden transmitir a los alumnos son, entre otras: recompensa, competición, reconocimiento o estatus, autoexpresión, altruismo, superación, etc. Las mecánicas también son muy variadas: avatares, insignias, puntos, colecciones, rankings, niveles, bienes virtuales, misiones y retos, desafíos, etc. (de Cea, 2014).

En buena parte, el éxito o el fracaso de las dinámicas gamificadas depende del grado de identificación del alumno con la dinámica y la actividad en sí misma. Según Valderrama (2015), hay que tener en cuenta el perfil del estudiante y programar la actividad en consecuencia: hedonistas / luchadores, exploradores / controladores, guerreros / constructores, socializadores / solitarios, etc. Personalizar los retos permite atender a diferentes ritmos de aprendizaje (Monguillot et al., 2015).

La actividad implica desafíos en todas sus etapas: diseño, ejecución y realimentación. Muchos trabajos publicados identifican como una gran ventaja de la gamificación la mejora del trabajo en grupo y la sociabilización de los alumnos: competir, colaborar y comparar logros (Gonzalez y Mora, 2015 y Riquel, 2014). Existen importantes dificultades para fijar objetivos, establecer roles, gestionar la dinámica, definir indicadores, etc. En muchas ocasiones se trata de un proceso de prueba y error. La satisfacción del alumnado y la medición de los objetivos son una buena guía para dirigir la actividad en los sucesivos cursos.

Existen numerosas herramientas que facilitan la gamificación de actividades. A continuación, se realiza una somera descripción de las más conocidas.

- Socrative: es una herramienta para conocer la respuesta de los alumnos en tiempo real mediante PCs y móviles. La aplicación es gratuita. Presenta una gran versatilidad en cuanto al formato de preguntas que se pueden plantear: de múltiple opción, de verdadero o falso, respuestas cortas, etc.

- QuizBean (Bluehouse Gruop Production, 2015) permite realizar exámenes interactivos.

- Proyecto Descartes (Proyecto Descartes, 2015), permite elaborar recursos didácticos interactivos que se embeben en páginas html.

- Kuizza (Kuizza, 2015) permite que los profesores creen un cuestionario y compartirlo con los alumnos.

En la bibliografía consultada, predomina la gamificación mediante juegos de cuestiones (Castilla et al., 2013 e Ibañez, 2015).

En el presente trabajo se describen algunas experiencias de gamificación que se están aplicando en la asignatura "Energy and Sustainable Development” del Máster Universitario de Ingeniería Industrial (MUII) de la Escuela Técnica Superior de Ingenieros Industriales (ETSII)

(cc) EY-NG-ND 2018, Universitat Politècnica de València 
de la Universitat Politècnica de València (UPV). Estas metodologías utilizadas de manera conjunta ofrecen un escenario idóneo para trabajar algunas competencias transversales.

\section{Objetivos}

Los objetivos fundamentales de esta innovación son potenciar la motivación, la concentración y el esfuerzo mediante la aplicación de técnicas de gamificación combinadas con aprendizaje basado en casos en la asignatura "Energy and Sustainable Development" del Máster Universitario de Ingeniería Industrial (MUII) de la Escuela Técnica Superior de Ingenieros Industriales (ETSII) de la Universitat Politècnica de València (UPV).

Para ello se va a presentar el diseño de las actividades, las herramientas utilizadas y la implementación en clase. Los resultados de la innovación docente, al estar en desarrollo, se basan en la realimentación inicial por parte del alumnado.

Por último, se presenta la forma en la que se ha integrado el trabajo relativo a algunas competencias transversales en el marco de actividades ludificadas.

\section{Desarrollo de la innovación}

\subsection{Energy and Sustainable Development}

La asignatura en la que se ha desarrollado la innovación docente es "Energy and Sustainable Development” del Máster Universitario de Ingeniería Industrial (MUII) de la Escuela Técnica Superior de Ingenieros Industriales (ETSII) de la Universitat Politècnica de València (UPV). Se trata de una asignatura optativa con 4,5 créditos (2,4 de teoría y 2,1 de prácticas) y docencia íntegramente en inglés. Tiene un enfoque eminentemente práctico y está fundamentalmente dirigida a alumnos de intercambio. El curso 2017-2018 es el primero en el que se está impartiendo docencia. En la asignatura se analizan aspectos del problema energético relacionados con el medio ambiente y la sostenibilidad. Las competencias específicas están relacionadas con temas de energías renovables, energía nuclear y aspectos sociales, económicos y ambientales de la generación de energía. Se intenta dar un enfoque europeo, intentando reducir en la medida de lo posible, los ejemplos relativos a España y potenciando los casos y ejemplos de otros países europeos. Además de las competencias específicas, se trabajan principalmente tres competencias transversales según el marco establecido en el proyecto institucional de competencias transversales UPV. Estas competencias son: "Responsabilidad ética, profesional y medioambiental", "Conocimiento de problemas contemporáneos" e "Instrumental específica”. La docencia se distribuye en clases presenciales de 1,5 horas, con dos sesiones por semana. En el presente curso hay 10 alumnos matriculados de diversas nacionalidades: Alemania, Suecia, Finlandia e Italia. El perfil de los alumnos es eminentemente técnico. Todos ellos son graduados: ingeniería química, ingeniería mecánica e ingeniería de la energía.

Debido al reducido número de alumnos, el carácter descriptivo de la asignatura y el perfil internacional de los mismos se ha optado por minimizar la clase magistral para potenciar otras

(cc) EY-NC-ND 2018, Universitat Politècnica de València

Congreso IN-RED (2018) 
metodologías activas a las que, por otro lado, ya están acostumbrados la mayoría de los alumnos de esta asignatura.

Este marco tan específico es, a priori, un escenario idóneo para experimentar metodologías activas de enseñanza-aprendizaje. Con el objetivo doble de trabajar competencias específicas y transversales se ha decidido realizar experiencias aplicando técnicas de gamificación y aprendizaje basado en casos.

Los contenidos de la asignatura se pueden clasificar en tres grandes bloques: energías renovables, aspectos económicos y aspectos medio ambientales.

\subsection{Planteamiento de la gamificación}

Partiendo de la idea de la gamificación y el aprendizaje basado en casos, se ha intentado buscar los contenidos de la asignatura que mejor podrían adaptarse a este tipo de dinámicas. Es cierto que la propia asignatura se presta a ello, debido fundamentalmente al tipo de contenidos y a la repercusión social, económica y medioambiental de dichos contenidos. En primer lugar, se ha realizado una revisión de los contenidos buscando aquellos que ofrezcan mayor número de grados de libertad a la hora de buscar herramientas y técnicas de gamificación. Tal y como se ha mencionado en el apartado anterior, los contenidos de la asignatura se clasifican en los bloques de energías renovables, aspectos económicos y aspectos medio ambientales. En el bloque de energías renovables se estudia la energía solar, la energía eólica, las energías del mar y la energía hidráulica. Además, este bloque incluye un tema de introducción al problema energético en el que se presentan las reservas y producciones actuales a nivel mundial y se señalan los escenarios y retos a medio y largo plazo. Desde un principio los profesores de la asignatura hemos sido conscientes de la oportunidad que representa trabajar con alumnos procedentes de diferentes países y hemos querido convertir esta situación en una ventaja de cara a plantear la asignatura.

Con esta idea en mente, se han planteado diversas actividades gamificadas en el bloque de energías renovables. La idea fundamental de la experiencia es realizar la planificación energética de un país imaginario al que se le incluye una serie de restricciones económicas y medioambientales. ¿Cómo se puede abastecer de energía eléctrica a un determinado país? ¿Qué implicaciones económicas, sociales y ambientales tiene la generación de energía? ¿Qué factores diferencian a un país de otro en este tema? ¿Es bueno focalizar toda la inversión en unas pocas fuentes de energía? ¿Qué ventajas e inconvenientes presenta cada una de las formas de energía? ¿Sólo renovables? ¿Y la nuclear? Esta batería de preguntas no tiene por objetivo abrumar al alumnado, sino hacerles partícipes de la complejidad del problema.

Los alumnos trabajan por parejas y todos parten de una "hoja en blanco" que caracteriza el país. El país en cuestión dispone de un presupuesto determinado para construir (desde cero) parques eólicos, plantas fotovoltaicas, centrales de biomasa, centrales hidroeléctricas, etc. Además del presupuesto de construcción, se proporciona un presupuesto anual para cubrir los gastos básicos del sistema (excepto los derivados de la venta de la energía eléctrica, que son sufragados por los consumidores del país). 
Los costes de inversión y de generación de estas diferentes tecnologías se han extraído del Plan de Energías Renovables (PER 2011-2020) del Ministerio de Energía, Turismo y Agenda Digital, de tal forma que se dispone del orden de magnitud relacionado con los costes de inversión y generación de cada fuente de energía.

Por otra parte, el sistema de generación de energía debe cubrir una demanda anual con variaciones diarias (con máximos en horas punta y mínimos de demanda en horas valle).

Así pues, las restricciones y condiciones del juego son las siguientes:

- Un país con una determinada demanda de energía eléctrica anual con máximos y mínimos diarios.

- Disponibilidad para construir cualquier instalación que utilice energías renovables.

- Disponibilidad de un máximo de capital de inversión.

- Se deberá tener en cuenta el coste de generación de la energía y aspectos medioambientales.

Una vez presentado el escenario, cada grupo deberá realizar la planificación del sistema energético de ese país teniendo en cuenta las restricciones enumeradas. La gamificación se extiende a lo largo de todo el curso, puesto que a medida que se presentan los contenidos teóricos de la asignatura, cada grupo aprende a estimar la energía que se produce con cada una de las tecnologías.

Al finalizar el curso, los equipos presentan su planificación energética. El grupo que mejor consigue adaptarse a la demanda, utilizando un mayor número de tecnologías con el menor coste de inversión y/o generación, gana la competición.

En una sesión final, los equipos presentan su planificación exponiendo los pros y contras de su propuesta y las implicaciones económicas y medioambientales.

La conclusión final del trabajo es que a partir de las condiciones presentadas no se puede garantizar la cobertura de la demanda a no ser que se compatibilice el uso de las renovables con otras tecnologías de carácter convencional como las centrales de carbón o las nucleares, porque no se va a poder cubrir la demanda en periodos en los que no existan buenas condiciones de viento, radiación solar, etc.

Así pues, el objetivo de la dinámica es doble. Por un lado, aprender a realizar cálculos de dimensionado de instalaciones eólicas, fotovoltaicas, de biomasa e hidráulicas y, por otro lado, hacer conscientes a los alumnos de la necesidad de compatibilizar el mayor número posible de tecnologías en aras de garantizar el consumo.

Tal y como se puede concluir, con las actividades propuestas se están trabajando diversas competencias transversales, como "Comunicación Efectiva” o "Conocimiento de Problemas Contemporáneos”, ya que, en la asignatura, el alumnado trabaja para analizar el problema del abastecimiento energético a las colectividades.

(c) ) EY-NC-ND 2018, Universitat Politècnica de València

Congreso IN-RED (2018) 


\subsection{Diseño de actividades}

A lo largo del curso, los equipos van aplicando los conocimientos adquiridos para determinar, con el presupuesto disponible, qué tecnologías van a utilizar, dónde se van a situar, cuál va a ser la energía que van a producir y cuál va a ser el coste, tanto de inversión como de generación.

A continuación, se describe someramente las actividades propuestas.

- Actividad 1: Energía eólica.

En esta actividad se establecen las bases para el cálculo y dimensionado de parque eólicos. Se explica el funcionamiento de la herramienta informática on-line “Atlas eólico” del Instituto para la Diversidad y Ahorro Energético (IDAE). Se explican los fundamentos de los cálculos eólicos y mediante el uso del "Atlas eólico" se plantea al grupo que busquen y seleccionen ubicaciones idóneas para la construcción de parques eólicos. Estas ubicaciones deben tener buenas características en cuanto a viento (densidad de potencia eólica) y en cuanto a características orográficas (disposición de cimas y cordilleras con direcciones apropiadas respecto a la dirección predominante del viento). En la Figura 1 se muestra, a modo de ejemplo, una zona concreta con sus características eólicas y orográficas.

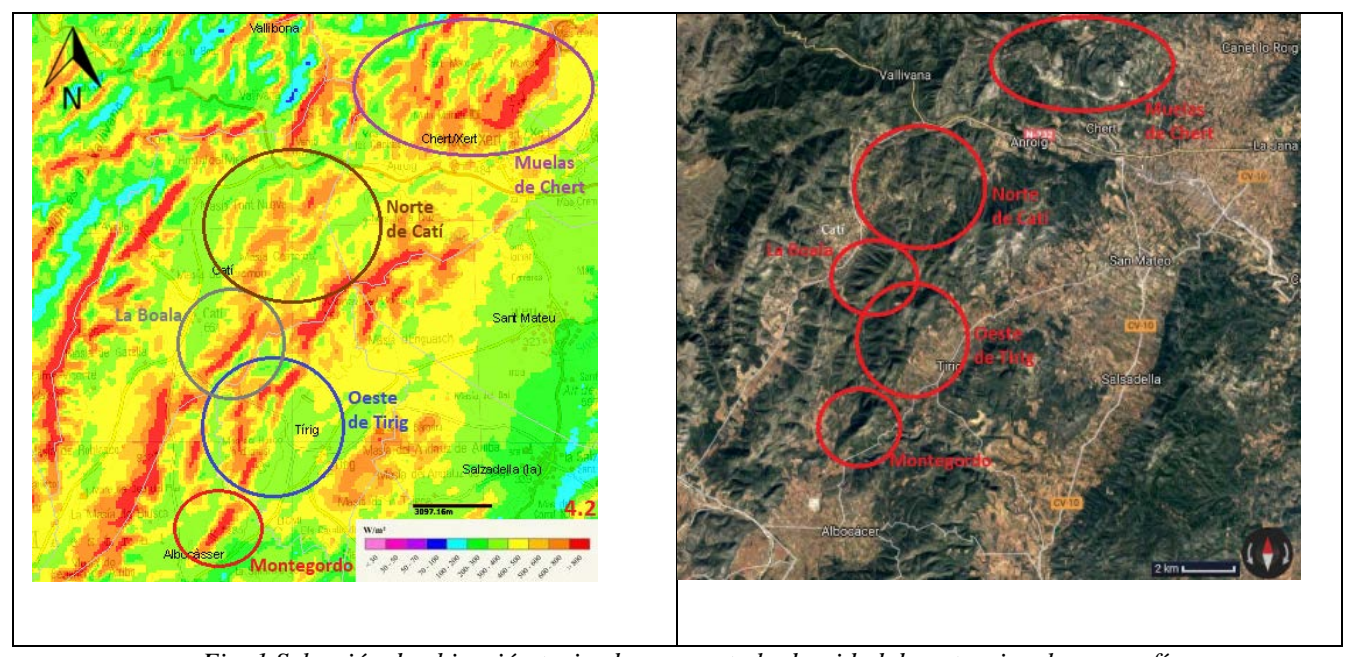

Fig. 1 Selección de ubicación teniendo en cuenta la densidad de potencia y la orografía.

Con la metodología explicada en la parte teórica, el alumnado aprende a estimar cuántos aerogeneradores y que energía podría producirse en una determinada ubicación. Con esta competencia específica adquirida, se utiliza el mapa eólico de España para que los alumnos localicen diferentes ubicaciones hasta alcanzar un máximo de potencia total.

- Actividad 2: Energía solar fotovoltaica.

Siguiendo la misma idea de la Actividad 1, se proporcionan las herramientas necesarias para calcular la energía que se puede producir mediante la tecnología solar fotovoltaica. Una vez

(c)) EY-NG-ND 2018, Universitat Politècnica de València 
expuestos los cálculos teóricos, se presenta la utilidad Photovoltaic Geographical Information Syste (PVGIS) de la Comisión Europea y se explica su uso. En la Figura 2 se muestra un cálculo correspondiente a una localización determinada en Italia. En este caso, los alumnos podrán buscar ubicaciones posibles para construir diferentes centrales fotovoltaicas hasta alcanzar un máximo de potencia instalada. El resultado del cálculo será, de nuevo, la energía capaz de producirse por dichas centrales.

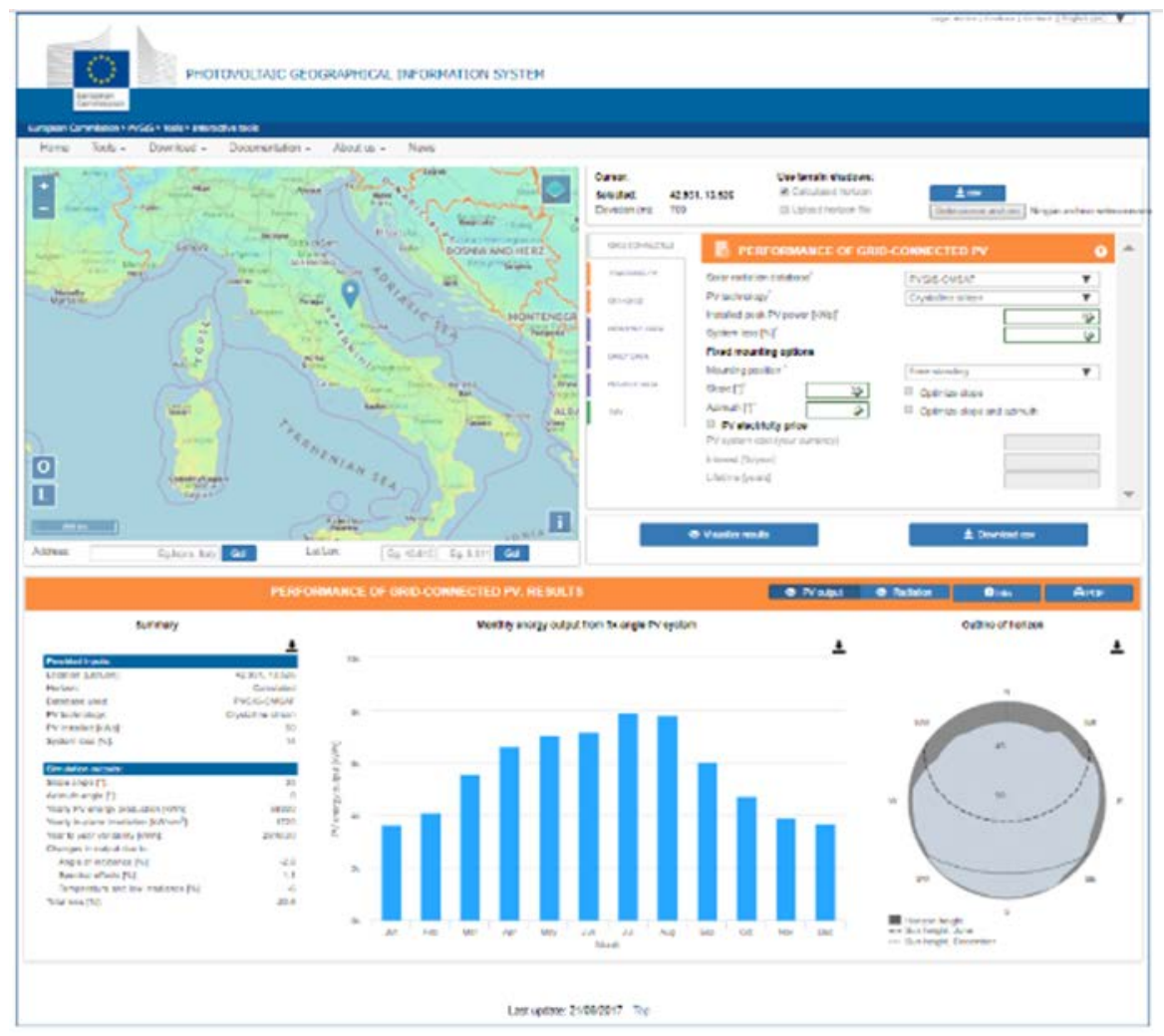

Fig. 2 Herramienta de cálculo PVGIS para estimar la energía eléctrica con fotovoltaica.

- Actividad 3: Energía hidroeléctrica.

Aplicando la misma metodología, se expone como se evalúa la producción de energía a partir del caudal de un río o de un volumen embalsado. Se utiliza el software Retscreen ( $)$ para estimar la producción energética de un determinado río, teniendo en cuenta limitaciones técnicas y medioambientales. Para el caso de aplicación del país imaginario, se facilitará el inventario de embalses de un país como España, con su producción eléctrica anual y la estimación del coste de construcción de una central genérica por MW instalado. 
- Actividad 4: Otras fuentes de energía. Energías del mar.

En este caso, debido al grado de inmadurez de las tecnologías, la exposición se limita a describir las posibles formas del aprovechamiento energético y a realizar un inventario de las instalaciones existentes a nivel mundial.

- Actividad 5. Cálculo económico.

Una vez descritas las tecnologías básicas se proporcionan las bases para realizar un cálculo económico, conociendo los costes de inversión, el coste de generación y una aproximación a las tarifas aplicables. El alumno aprende a calcular parámetros económicos como la Tasa Interna de Rentabilidad, el Valor Actual Neto y el Periodo de Retorno. Estos parámetros van a ser muy importantes para que cada grupo evalúe la rentabilidad y sostenibilidad económica de las soluciones que ellos mismos propongan.

- Actividad 6. Impacto ambiental.

Por último, se proporcionan unas directrices básicas de cómo se realiza una evaluación de impacto ambiental, qué proyectos deben someterse a tal estudio y cómo se establece un plan de prevención y minimización de impactos.

El objetivo la gamificación propuesta es obtener puntos o “estrellas” por retos. Cada tecnología (o actividad) representa un reto. Obtiene mayor puntuación en cada reto el grupo que cumple el mayor número de los siguientes criterios:

- Mayor cantidad de energía al menor coste de inversión (MW/€). Máximo 10 estrellas.

- Calidad de los resultados en cuanto a selección de ubicaciones, cálculos razonados y calidad de las fuentes de información consultadas. Máximo 10 estrellas.

En las Actividades 1, 2 y 3, se evalúan los dos criterios, y se van acumulando el número de estrellas. Las Actividades 4, 5 y 6 se puntúan mediante cuestionarios Socrative con un máximo de 10 estrellas por actividad.

En la puesta en común se pueden conseguir 20 estrellas adicionales. Esta puntuación se reparte de la siguiente forma:

- 10 estrellas por la puntuación de los profesores, atendiendo a criterios de razonamiento de los alumnos en cuanto a la idoneidad de la planificación propuesta por los equipos. ¿Se trata de una buena solución? ¿Se llega a cubrir la demanda del país? ¿Cómo se han tenido en cuenta la variabilidad de la demanda?

- 10 estrellas por la puntuación de los restos de equipos. Para ello, se facilita un pequeño cuestionario para que valoren en función de una serie de ítems, la actuación de los demás equipos.

(c) 2018, Universitat Politècnica de València 
Al finalizar la dinámica, el profesor valora el trabajo global realizado en las diferentes actividades, la capacidad de razonamiento, la disposición a entablar un debate aportando o rebatiendo argumentos, la calidad de la exposición y del trabajo en general.

\subsection{Competencias transversales}

Con esta metodología se trabajan algunas competencias transversales, fundamentales para el perfil profesional y formativo de los alumnos. Concretamente: "Conocimiento de problemas contemporáneos" permite tomar conciencia de nuestro entorno, facilitando la propuesta de soluciones a los problemas del campo profesional; "Responsabilidad ética, profesional y medioambiental” fomenta el actuar de un modo coherente y la responsabilidad en la actuación y toma de decisiones, así como la capacidad para juzgar actuaciones profesionales en términos de respeto a la sociedad y al medio ambiente. Por último, la competencia "Instrumental específica" ayuda a dominar las herramientas específicas.

\section{Resultados}

\subsection{Evaluación de la materia específica}

A partir de esta aplicación de la gamificación se evalúan varios contenidos de la materia específica de la asignatura como el diseño de sistemas, cálculos económicos y la consideración de aspectos ambientales en los proyectos de ingeniería, así como la asimilación de éstos en su argumentación y discusión. Esto se realiza a partir de diversos criterios. Mediante la puntuación subjetiva de los compañeros y del profesor en el debate, así como la puntuación obtenida en los cuestionarios Socrative y el ratio MW/€ en la gamificación, se obtiene una valoración global.

De este modo, la calificación puede ser proporcional al número de "estrellas" obtenido en el “juego”. La valoración última representa un $25 \%$ de la nota final de la asignatura en concepto de trabajos académicos y exposiciones.

\subsection{Evaluación de competencias transversales}

En cuanto a las competencias transversales trabajadas, se presenta a modo de ejemplo en la Tabla 1, la evaluación correspondiente a la competencia "Conocimiento de Problemas Contemporáneos”. Al tratarse de una asignatura de máster, se ha seguido un nivel de dominio III.

A partir de los indicadores listados en la Tabla 1, se califica los resultados obtenidos en una escala de valoración de cuatro valores $A, B, C, D$, correspondiéndose a: excelente (A), adecuado (B), en desarrollo (C) o no alcanzado (D). 
Tabla 1. Rúbrica para "Energy and Sustainable Development” - Nivel de dominio III-

\begin{tabular}{|c|c|c|c|c|}
\hline Indicador & $\begin{array}{ll}\text { D. } & \text { No } \\
\text { alcanzado } & \end{array}$ & C. En desarrollo & $\begin{array}{l}\text { B. Bien } \\
\text { /adecuado }\end{array}$ & $\begin{array}{l}\text { A. Excelente } \\
\text { Ejemplar }\end{array}$ \\
\hline $\begin{array}{l}\text { Analiza y relaciona aspectos sociales } \\
\text { se ven afectados por el abastecimiento } \\
\text { de energía a las colectividades. }\end{array}$ & $\begin{array}{ll}\text { No } & \text { analiza } \\
\text { ningún } & \text { aspecto } \\
\text { social. } & \end{array}$ & $\begin{array}{l}\text { Analiza sin } \\
\text { relacionar uno o } \\
\text { dos problemas } \\
\text { sociales. }\end{array}$ & $\begin{array}{l}\text { Analiza y } \\
\text { relaciona uno o } \\
\text { dos problemas } \\
\text { sociales. }\end{array}$ & $\begin{array}{lr}\text { Analiza } & \text { y } \\
\text { relaciona } & \text { más } \\
\text { de } & \text { dos } \\
\text { problemas } & \\
\text { sociales. } & \end{array}$ \\
\hline $\begin{array}{l}\text { Analiza y relaciona aspectos } \\
\text { medioambientales se ven afectados } \\
\text { por el abastecimiento de energía a las } \\
\text { colectividades. }\end{array}$ & $\begin{array}{l}\text { No analiza } \\
\text { ningún medio } \\
\text { ambiental. }\end{array}$ & $\begin{array}{l}\text { Analiza sin } \\
\text { relacionar uno o } \\
\text { dos problemas } \\
\text { medio } \\
\text { ambientales. }\end{array}$ & $\begin{array}{l}\text { Analiza y } \\
\text { relaciona uno o } \\
\text { dos problemas } \\
\text { medio } \\
\text { ambientales. }\end{array}$ & $\begin{array}{lr}\begin{array}{l}\text { Analiza } \\
\text { relaciona }\end{array} \text { más } \\
\text { de } & \text { dos } \\
\text { problemas } & \\
\text { medio } & \\
\text { ambientales. }\end{array}$ \\
\hline $\begin{array}{l}\text { Analiza y relaciona las repercusiones } \\
\text { económicas del abastecimiento de } \\
\text { energía a las colectividades. }\end{array}$ & $\begin{array}{l}\text { No analiza } \\
\text { ninguna } \\
\text { repercusión } \\
\text { económica. }\end{array}$ & $\begin{array}{l}\text { Analiza sin } \\
\text { relacionar una o } \\
\text { dos } \\
\text { repercusiones } \\
\text { económicas. }\end{array}$ & $\begin{array}{l}\text { Analiza y } \\
\text { relaciona una o } \\
\text { dos } \\
\text { repercusiones } \\
\text { económicas. }\end{array}$ & $\begin{array}{l}\text { Analiza y } \\
\text { relaciona más } \\
\text { de dos } \\
\text { repercusiones } \\
\text { económicas. }\end{array}$ \\
\hline
\end{tabular}

La gamificación se presta al trabajo de esta competencia porque durante toda la asignatura se realizan continuas referencias a las implicaciones sociales, medio ambientales y económicas del problema energético. Se utiliza Socrative para introducir cuestiones relacionadas con esta competencia durante la gamificación. La rúbrica de evaluación se aplica para objetivar el desempeño de los grupos en esta competencia y se utiliza durante la presentación que se realiza al finalizar la dinámica de gamificación.

\subsection{Evaluación de la metodología}

Debido al gran nivel de compromiso e implicación de los estudiantes al asumir el rol de profesional de la ingeniería, gracias al carácter realista de esta actividad, se ha apreciado una comprensión más profunda de los conceptos de la asignatura, lo que les ha permitido aplicarlos de forma correcta a las actividades. Para que esta metodología pueda desarrollarse correctamente por parte de los alumnos, habida cuenta que se emplean diferente software para los cálculos de las instalaciones que diseñan, es de gran importancia que el profesor que imparte las clases prácticas en las que se enseña el manejo de los programas, constate que se han adquirido los conocimientos necesarios para realizar los cálculos de forma autónoma. Asimismo, dado que preparan y realizan una exposición final del trabajo que han llevado a cabo, se mejora y potencia su expresión oral y dotes comunicativas, maximizándose la participación al trabajar en grupos pequeños.

Actualmente, todavía no se dispone de información relativa a la satisfacción del alumnado por la metodología utilizada, aunque si que se dispone de una realimentación informal a partir de charlas con los alumnos. La mayoría reconoce la utilidad de esta metodología siempre y cuando se compagine con otras técnicas (estudio de casos, trabajos por proyectos, etc.).

(c) B BY-NC-ND 2018, Universitat Politècnica de València

Congreso IN-RED (2018) 


\section{Conclusiones}

En los últimos años se ha visto la importancia de diseñar unas clases donde los alumnos tengan un rol más activo. Con este fin, se pensó en la metodología de la gamificación, que actualmente se está aplicando en la asignatura "Energy and Sustainable Development" del Máster Universitario de Ingeniería Industrial (MUII) de la Escuela Técnica Superior de Ingenieros Industriales (ETSII) de la Universitat Politècnica de València (UPV). Este trabajo presenta la experiencia de la implantación de dicha técnica.

Las actividades que se llevan a cabo combinan la metodología de la gamificación con el aprendizaje basado en casos. El objetivo de la dinámica es doble: por un lado, aprender a realizar cálculos de dimensionado de instalaciones eólicas, fotovoltaicas, de biomasa e hidráulicas y, por otro, hacer consciente al alumnado de la necesidad de compatibilizar el mayor número posible de tecnologías en aras de garantizar la demanda. Se premia mediante “estrellas” la obtención de unos resultados satisfactorios.

A su finalización, se defiende el trabajo ante el grupo-clase. El profesor debe valorar el trabajo global llevado a cabo por el alumno, su capacidad de razonamiento, la disposición a entablar un debate aportando o rebatiendo argumentos, la calidad de la exposición y del trabajo en general. También interaccionan el resto de los compañeros, que valoran la actuación de los demás equipos.

Con esta metodología se pretende que el alumno adquiera conocimientos partiendo de las conclusiones que adquiere de su propio razonamiento. El éxito radica, en gran medida, en su motivación.

\section{Agradecimientos}

Este trabajo se ha realizado en el marco del proyecto Proyecto de Innovación y Mejora Educativa, PIME Curso 2017-2018 "Coordinación de competencias transversales en asignaturas de ámbito nuclear en el Grado de Ingeniero de la Energía”, Referencia B07, del Vicerrectorado de Estudios, Calidad y Acreditación de la Universitat Politècnica de València.

\section{Referencias}

ROMERO, M. y PÉREZ FERRA, M. (2009). “Cómo motivar a aprender en la universidad: una estrategia fundamental contra el fracaso académico en los nuevos modelos Educativos". Revista Iberoamericana de Educación, vol. 51, p. 87-105.

ATTALI, Y. y ARIELI-ATTALI, M. (2015) "Gamification in assessment: Do points affect test performance?”, Computers \& Education, vol. 83, p. 57-63.

BRUDER, P. (2015). “GAME ON: Gamification in the Classroom”. Education Digest, vol. 80 (7), p. 56-60.

CASTILlA, G., ROMANA, M. G. y LOPEZ-TERRADAS, B. (2013) "Concursando en el aula: la gamificación mediante quiz-show como herramienta de dinamización docente” en : $X$ 
Jornadas Internacionales de Innovación Universitaria. Educar para transformar. Universidad Europea.

CEA, A. (2014) "Diseño y desarrollo de aplicaciones software para la creación de actividades docentes con elementos de Gamificación”. Universidad Autónoma ed Madrid.

DETERDING, et al. (2011). “Gamification: Toward a Definition” en CHI 2011. Vancouver. AMC 978-1-4503-0268-5/11/05.

GONZALEZ, C.S. y MORA, A. (2015). “Técnicas de gamificación aplicadas en la docencia de Ingeniería Informática”. Revista de Investigación en Docencia Universitaria de la Informática (ReVisión) vol 8, núm 1.

IBAÑEZ MORENO, A. (2015). La gamificación para (auto)evaluar las competencias léxicogramaticales en el aula de inglés como segunda lengua en el contexto de la enseñanza a distancia: un estudio de caso. Verbeia Número 0. Universidad Camilo José Cela.

KAPP, K. M. (2012). The gamification of learning and instruction: Game-based methods and strategies for training and education. San Francisco: Pfeiffer.

LLAGOSTERA, E. (2012). “On Gamification and Persuasion” en XI SB Game. SBCProceeding of SB Games. Brasilia. Games for change-Full papers. p.12-21.

MONGUILLOT HERNANDO, M. et al. (2015) "Play the Game: gamificación y hábitos saludables en educación física”. Apunts. Educación Física y Deportes vol. 119, p. 71-79.

RIQUEL, A.M., (2014) "Incrementando la motivación en la clase de ELE: el uso de la gamificación en el aula”. Universidad de Sevilla.

URQUIDI MARTIN, A. C. y CALABOR PRIETO, M. S. (2014) “Aprendizaje a través de juegos de simulación: un estudio de los factores que determinan su eficacia pedagógica”. EDUTEC. Revista Electrónica de Tecnología Educativa.

VALDERRAMA, B. (2015) “Los secretos de la gamificación: 10 motivos para jugar”. Capital Humano vol. 295, p.72-78.

WERBACH, K. (2014). “(Re) Defining Gamification: A Process Approach” Spagnolli, A. en Persuasive Technology. PERSUASIVE 2014. Lecture Notes in Computer Science. Springer International Publishing. vol. 8462, p. 266-272. 